\title{
Resistência de união na cervical de cavidades classe II com resinas Bulk Fill
}

\author{
Cervical bond strength of class II cavities with Bullk Fill resins
}

Resistencia de la unión cervical de las caries de clase II con resinas Bulk Fill

\section{Resumo}

Objetivo: O objetivo deste estudo foi avaliar in vitro a resistência de união através do teste de microtração de resinas compostas bulk fill do tipo flow à dentina da margem cervical de cavidades classe II de molares humanos recém extraídos. Metodologia: Foram padronizadas 50 cavidades com $4 \mathrm{~mm}$ de profundidade e $3 \mathrm{~mm}$ de largura vestíbulolingual, na mesial e distal de 25 molares. As cavidades $(n=10)$ foram restauradas com as resinas: Filtek Z350XT e Filtek Flow (3M/ESPE); Filtek Bulk Fill Flow (3M/ESPE); Surefill SDR Flow (Dentsply) e X-tra Base (Voco). Após $24 \mathrm{~h}$ em estufa $\left(37^{\circ} \mathrm{C}\right)$, as amostras foram seccionados em palitos da parede cervical das caixas proximais, com uma área e seção transversal de $\pm 1 \mathrm{~mm}^{2}$ e tracionados em uma máquina de ensaio universal (Kratos/modelo IKCL3-USB), velocidade de $1 \mathrm{~mm} / \mathrm{min}$. $\mathrm{O}$ dados obtidos em MPa foram submetidos à teste estatístico ANOVA e Tukey $(\mathrm{p}<0.005)$. A média da resistência de união mais elevada foi registrada no grupo SDR (64.37 MPa), seguida dos grupos X-tra Base (41.46 MPa), Filtek Flow (36.85 MPa), Filtek Bulk Fill Flow (26.98Mpa) e Filtek Z350 (20.99 MPa). Conclusão: As resinas bulk fill de baixa viscosidade estudadas demonstraram resistências de união satisfatória nas paredes cervicais de cavidades classe II.

Palavras-chave: Compósitos; Resina Bulk Fill; Microtração; Tensão de polimerização.

\begin{abstract}
Objective: The aim of this study was to evaluate in vitro the bond strength through the microtensile test of flow-type bulk fill composite resins to the dentin of the cervical margin of class II cavities of newly extracted human molars. Methodology: Fifty cavities $4 \mathrm{~mm}$ deep and $3 \mathrm{~mm}$ wide buccolingually, mesial and distal, of 25 molars were standardized. The cavities $(\mathrm{n}=10)$ were restored with the following resins: Filtek Z350XT and Filtek Flow (3M/ESPE); Filtek Bulk Fill Flow (3M/ESPE); Surefill SDR Flow (Dentsply) and X-tra Base (Voco). After 24 hours in an oven $\left(37^{\circ} \mathrm{C}\right)$, the samples were sectioned on sticks from the cervical wall of the proximal boxes, with an area and cross section of $\pm 1 \mathrm{~mm} 2$ and pulled in a universal testing machine (Kratos/model IKCL3-USB), speed of $1 \mathrm{~mm} / \mathrm{min}$. Data obtained in MPa were submitted to ANOVA and Tukey statistical tests $(\mathrm{p}<0.005)$. The mean of the highest bond strength was recorded in the SDR group (64.37 MPa), followed by the X-tra Base (41.46 MPa), Filtek Flow (36.85 $\mathrm{MPa}$ ), Filtek Bulk Fill Flow (26.98Mpa) and Filtek Z350 (20.99 MPa) groups. MPa). Conclusion: The low viscosity bulk fill resins studied showed satisfactory bond strengths on the cervical walls of class II cavities.
\end{abstract}

Keywords: Composites; Bulk Fill resin; Microtraction; Polymerization stress.

\section{Resumen}

Objetivo: El objetivo de este estudio fue evaluar in vitro la fuerza de unión a través de la prueba de microtensión de resinas compuestas de relleno a granel tipo flow a la dentina del margen cervical de cavidades clase II de molares humanos recién extraídos. Metodología: Se estandarizaron 50 cavidades de $4 \mathrm{~mm}$ de profundidad y $3 \mathrm{~mm}$ de ancho vestibular, mesial y distal, de 25 molares. Las cavidades $(n=10)$ se restauraron con las siguientes resinas: Filtek Z350XT y Filtek Flow (3M / ESPE); Flujo de llenado a granel Filtek (3M / ESPE); Surefill SDR Flow (Dentsply) y $\mathrm{X}$-tra Base (Voco). Después de 24 horas en horno $\left(37^{\circ} \mathrm{C}\right)$, las muestras se seccionaron en varillas de la pared cervical 
de las cajas proximales, con un área y sección transversal de $\pm 1 \mathrm{~mm} 2$ y se tiraron en una máquina de prueba universal (Kratos / modelo IKCL3-USB), velocidad de $1 \mathrm{~mm} / \mathrm{min}$. Los datos obtenidos en MPa se sometieron a ANOVA y pruebas estadísticas de Tukey ( $\mathrm{p}<0,005)$. La media de la fuerza de unión más alta se registró en el grupo SDR $(64,37$ MPa), seguido por X-tra Base (41,46 MPa), Filtek Flow (36,85 MPa), Filtek Bulk Fill Flow (26,98 Mpa) y Filtek Z350 (20,99 MPa) grupos MPa). Conclusión: Las resinas de relleno a granel de baja viscosidad estudiadas mostraron resistencias de adherencia satisfactorias en las paredes cervicales de las cavidades de clase II.

Palabras clave: Composites; Resinas de relleno a granel; Microtensiles; Estrés de polimerización.

\section{Introdução}

Um dos desafios da odontologia restauradora é encontrar o equilíbrio entre protocolos clínicos simples para restaurações com resinas compostas e longevidade clínica das restaurações (Yazici et al., 2017). Há uma variedade de compósitos resinosos que se diferenciam de acordo com os tipos, tamanho, peso e volume das partículas de carga, sendo classificados quanto à sua viscosidade, em resina do tipo flow (baixa viscosidade) e resina restauradora (monômeros viscosidade regular, passível de escultura) e quanto à técnica de inserção em resinas convencionais, (inseridas por meio da técnica incremental), e resinas bulk, (inseridas em incremento único de até $5 \mathrm{~mm}$ ), sendo uma alternativa para restaurações em dentes posteriores (Durão et al., 2020; Ferracane, 2011; van Ende et al., 2017).

A técnica incremental assegura melhor adaptação marginal, fotoativação mais efetiva, garantindo propriedades físicas adequadas (Park et al., 2008; Rosatto et al., 2015; Yazici et al., 2017). Porém, também há maior preocupação desta técnica restauradora, devido a possível contaminação e/ou incorporação de ar entre os incrementos de resina, promovendo uma adesão inadequada, tempo clínico mais longo (El-Safty et al., 2012; Lazarchik et al., 2007).

As resinas bulk fill permitem inserção em incremento único contribuindo na redução do tempo clínico e chances de contaminação ou incorporação de ar durante sua inserção (Chesterman et al., 2017; Li et al., 2015). Inserção em maior espessura é possível devido a maior translucidez de sua matriz orgânica, permitindo maior profundidade de cura e là presença de monômero modulador de contração, que interfere na qualidade adesiva ao substrato dental, e nas tensões geradas durante sua contração de polimerização, característica inerente do material (N. Ilie et al., 2013; Rosatto et al., 2015; van Dijken \& Pallesen, 2015; Van Dijken \& Pallesen, 2014; Zaruba et al., 2013). Sua resistência à compressão e à tração, módulo de elasticidade, contração da polimerização, tensão de contração e grau de conversão desempenham papéis significativos no sucesso clínico (van Dijken \& Pallesen, 2015).

No entanto, a qualidade da adesão na região marginal, principalmente na parede cervical da caixa proximal é mais crítica e pode ocasionar degradação marginal, micro infiltração, perda precoce da restauração, sensibilidade pós-operatória e até mesmo injúria pulpar (NASCIMENTO et al., 2016). Visando contribuir com os profissionais no conhecimento e escolha das resinas bulk como material restaurador. O objetivo desse estudo foi avaliar e comparar a resistência de união em restaurações com resinas compostas bulk fill do tipo flow, com resinas convencionais de viscosidade baixa e regular, tendo como hipótese nula, que as resinas compostas estudadas não apresentam diferenças significativas.

\section{Metodologia}

Tipo de estudo

Se trata de um estudo do tipo experimental laboratorial in vitro.

\section{Local do estudo}

O estudo foi desenvolvido no Centro de Pesquisa em Biomateriais ( $\mathrm{CPqB}$ ) da Faculdade de Odontologia da Universidade de Pernambuco (FOP/UPE) e no laboratório multidisciplinar da Uninassau, Unidade Graças, Recife/PÉ. 


\section{Considerações Éticas}

Este estudo foi aprovado pelo Comitê de Ética em Pesquisa Envolvendo Seres Humanos da Universidade de Pernambuco, Brasil (parecer $\mathrm{n}^{\circ}$ 4.535.940).

\section{Seleção e armazenamento dos dentes}

Como critérios de inclusão: dentes terceiros molares humanos hígidos, extraídos por indicação terapêutica há cerca de 8 meses antes do início deste estudo, livres de trincas, fissuras ou rachaduras. Os critérios de exclusão foram dentes cariados, dentes com manchas hipoplásicas, com desgaste oclusal. Foram utilizados 25 molares, armazenados em solução aquosa de timol a $0,1 \%$ durante 24 horas para desinfecção. Em seguida lavados em água corrente, limpos com curetas periodontais e realizada profilaxia com pedra pomes e água, sendo armazenados em água destilada, substituída semanalmente, em estufa biológica $37^{\circ} \mathrm{C}$.

Os dentes tiveram sua porção oclusal removida através de um disco diamantado (Buehler, Illinois, EUA), montado em cortadeira de precisão Elsaw (Elquip, São Paulo, Brasil), sob refrigeração, a fim de planificá-la e facilitar a padronização das cavidades.

\section{Preparos Cavitários}

Cinquenta cavidades classe II tipo slot vertical foram padronizadas, preparadas nas faces mesial e distal dos 25 molares, com a parede gengival localizada 0,5 - 1,0mm abaixo da junção amelodentinária. As dimensões das cavidades padronizadas com $4 \mathrm{~mm}$ de profundidade e $3 \mathrm{~mm}$ de largura vestíbulo-lingual e axial, utilizando-se uma ponta diamantada cilíndrica de extremidade plana n..$^{\circ}$ 2096, 3 mm de diâmetro e 6 mm de comprimento de parte ativa (KG Sorensen, Cotia-SP, Brasil) acoplada a uma máquina de preparo automática (MPC auto, ElQuip, Ribeirão Preto-SP, Brasil) sob refrigeração água/ar constante. Uma relação de 01 ponta diamantada para cada 05 preparos foi respeitada. As cavidades foram avaliadas utilizando lupa estereoscópica a 15x de ampliação para verificar a presença de defeitos. (Nayif et al., 2008)

A seguir, os 25 dentes foram aleatoriamente divididos em 5 grupos, cada um com 5 dentes, totalizando 10 cavidades $(\mathrm{n}=10)$. Estas foram restauradas com Sistema Adesivo Scotchbond Universal (3M ESPE- St. Paul, MN, USA), em seguida preenchidas com os compósitos conforme suas referidas técnicas e divisão dos grupos descritos na Tabela 1, seguindo as recomendações do fabricante, ressaltando que as resinas Filtek ${ }^{\mathrm{TM}} \mathrm{Z} 350$ e Filtek $^{\mathrm{TM}}$ Flow foram utilizadas como grupo controle. 
Tabela 1. Materiais, Grupos, composição, aplicação e fabricantes.

\begin{tabular}{|c|c|c|c|}
\hline Material & Composição & Aplicação & Fabricante \\
\hline $\begin{array}{l}\text { Surefil SDR }{ }^{\mathrm{TM}} \\
\text { Flow } \\
\text { Cor: U } \\
\text { Grupo: SDR }\end{array}$ & $\begin{array}{l}\text { Vidro de bário boro flúor alumínio silicato, } \\
\text { vidro de estrôncio alumínio flúor silicato; } \\
\text { Resina dimetacrilato uretano modificada; } \\
\text { bisfenol A dimetacrilato etoxilado } \\
\text { (EBPADMA); trietilenoglicol dimetacrilato } \\
\text { (TEGDMA); canforoquinona; butil hidroxi } \\
\text { tolueno; estabilizantes UV; dióxido de titânio; } \\
\text { pigmentos de óxido de ferro. }\end{array}$ & $\begin{array}{l}\text { Incremento único de } \\
\text { até } 4 \mathrm{~mm} \text {, } \\
\text { fotoativzxar por } 20 \mathrm{~s} \\
\text { pela oclusal }\end{array}$ & $\begin{array}{l}\text { DENTSPLY } \\
\text { DeTrey;Konst } \\
\text { az, Germany }\end{array}$ \\
\hline $\begin{array}{l}\text { Filtek }^{\mathrm{TM}} \text { Bulk Fill } \\
\text { Flow } \\
\text { Cor: A2 } \\
\text { Grupo: FBF }\end{array}$ & $\begin{array}{l}\text { Bis-GMA, Bis-EMA, UDMA, Procrylat. } \\
\text { Partículas de zircônia-sílica, trifluoreto de } \\
\text { itérbio. \% em peso/volume (carga): } 64,5 \text { / } \\
\text { 42,5. Tamanho da partícula: Nanohíbrida. }\end{array}$ & $\begin{array}{l}\text { Incremento único de } \\
\text { até } 5 \mathrm{~mm} \text {, fotoativar } \\
\text { por } 10 \mathrm{~s} \text { pela oclusal, }\end{array}$ & $\begin{array}{l}\text { 3M ESPE, St. } \\
\text { Paul, MN, } \\
\text { USA }\end{array}$ \\
\hline $\begin{array}{l}\text { X-tra base } \\
\text { Cor: A2 } \\
\text { Grupo: } \\
\text { XTRABASE }\end{array}$ & $\begin{array}{l}\text { Bis-GMA, UDMA, TEGDMA Partículas de } \\
\text { carga não informado pelo fabricante }(75 \% \text { em } \\
\text { carga inorgânica) }\end{array}$ & $\begin{array}{l}\text { Incremento único de } \\
\text { até } 4 \mathrm{~mm} \text {, fotoativar } \\
\text { por } 10 \mathrm{~s} \text {, pela oclusal }\end{array}$ & $\begin{array}{l}\text { VOCO } \\
\text { Cuxhaven, } \\
\text { Germany }\end{array}$ \\
\hline $\begin{array}{l}\text { Filtek }^{\mathrm{TM}} \mathrm{Z} 350 \\
\text { Cor:A2 } \\
\text { (Controle } \\
\text { viscosidade } \\
\text { regular) } \\
\text { Grupo: Z350 }\end{array}$ & $\begin{array}{l}\text { Bis-GMA, UDMA e Bis-EMA. Partículas de } \\
\text { zircônia-sílica. \% em peso/volume (carga): } \\
\text { 82/60. Tamanho da partícula: } \\
\text { Nanopartículada. }\end{array}$ & $\begin{array}{l}\text { Técnica incremental } \\
\text { de } 2 \mathrm{~mm} \text { em } 2 \mathrm{~mm} \text { e } \\
\text { fotoativar por } 20 \mathrm{~s} \\
\text { cada incremento. }\end{array}$ & $\begin{array}{l}\text { 3M ESPE St. } \\
\text { Paul, MN, } \\
\text { USA }\end{array}$ \\
\hline $\begin{array}{l}\text { Filtek }^{\mathrm{TM}} \text { Flow } \\
\text { Cor:A2 } \\
\text { (Controle } \\
\text { viscosidade } \\
\text { fluida) } \\
\text { Grupo: FFLOW }\end{array}$ & $\begin{array}{l}\text { Cerâmica silanizada tratada Dimetacrilato } \\
\text { substituída Bisfenol A diglicidil éter } \\
\text { dimetacrilato (BisGMA) Sílica tratada de } \\
\text { silano Dimetacrilato de trietilenoglicol } \\
\text { (TEGDMA) Fluoreto de itérbio (YbF3) } \\
\text { Polímero policaprolactona reagida Etil } \\
\text { 4-dimetil aminobenzoato (EDMAB) } \\
\text { Benzotriazol Difeniliodônio } \\
\text { hexafluorofosfato. }\end{array}$ & $\begin{array}{l}\text { Técnica incremental } \\
\text { de } 2 \text { em } 2 \mathrm{~mm}, \\
\text { fotoativar por } 20 \mathrm{~s} \\
\text { cada incremento. }\end{array}$ & $\begin{array}{l}\text { 3M ESPE St. } \\
\text { Paul, MN, } \\
\text { USA }\end{array}$ \\
\hline $\begin{array}{l}\text { Scotchbond } \\
\text { Universal } \\
\text { Adhesive }\end{array}$ & $\begin{array}{l}\text { BisGMA, MDP, Copolímero do Vitrebond }{ }^{\mathrm{TM}} \text {, } \\
\text { HEMA, Etanol,Água, Partículas de carga, } \\
\text { Silano, Iniciadores. }\end{array}$ & $\begin{array}{l}\text { Condicionamento } \\
\text { seletivo do esmalte } \\
\text { por } 30 \text { s. Aplicar uma } \\
\text { camada do adesivo de } \\
\text { forma ativa por } 20 \mathrm{~s}, \\
\text { secar por } 5 \mathrm{~s} \text { e } \\
\text { fotoativar por } 10 \mathrm{~s} \text {. }\end{array}$ & $\begin{array}{l}\text { 3M ESPE St. } \\
\text { Paul, MN, } \\
\text { USA }\end{array}$ \\
\hline
\end{tabular}

Bis-GMA - Bisfenil glicidil metacrilato, UDMA - Uretano dimetacrilato, Bis-MA - Bisfenol etil metacrilato, TEGDMA - Trietileno glicol dimetarilato, EBPDMA - etoxilado de bisfenol-A-dimetacrilato.

Fonte: Autores.

\section{Materiais}

Os incrementos das resinas flow foram inseridos nas cavidades utilizando-se ponteiras aplicadoras que acompanham os produtos, após adaptação de matriz metálica. Sendo iniciado, assim, o procedimento restaurador previamente já descrito. 
Para fotoativação foi utilizado aparelho de luz LED com irradiância de 1200 mW/cm² (Optilight Max / Gnatus, Ribeirão Preto, São Paulo, Brasil), sendo a fonte de luz aplicada na parte superior da cavidade (oclusal). Após o término das restaurações, os grupos foram novamente mantidos em água destilada e receberam acabamento nas proximais com tiras de lixa (3M ESPE, St. Paul, MN, USA), discos Sof-Lex Pop-On (3M ESPE, St. Paul, MN, USA).

Os espécimes foram armazenados em recipientes plásticos com água destilada pelo período de 24 horas, mantidos em estufa biológica, à temperatura de $37^{\circ} \mathrm{C}$.

\section{Preparo das Espécimes para Teste de Microtração}

As superfícies oclusal e proximal da restauração classe II foram destacadas com caneta permanente de cor preta para melhor visualização e identificação dos palitos. Em seguida, os dentes foram fixados na máquina de cortes seriados Elsaw (Elquip, São Paulo, Brasil) e cortados paralelamente ao seu longo eixo, em espessuras de 1,0 $\mathrm{mm} \pm 0,1 \mathrm{~mm}$, nos sentidos vestíbulo-lingual e mesio-distal, aferidos com auxílio de paquímetro digital (Mitutoyo; Tokyo, Japan), assim como a espessura do disco diamantado utilizado aferido em 0,3mm, o que também foi considerada, respeitando-se uma distância de 1,0 mm entre eles, com o objetivo de se obter fatias com $0,7 \mathrm{~mm}^{2}$ de espessura (descontado $0,3 \mathrm{~mm}$ da espessura do disco diamantado).

Cortes realizados, então, a base dos conjuntos foi seccionada perpendicularmente ao longo eixo, com auxílio do disco de diamantado (Buehler, Illinois, EUA). Vale ressaltar que palitos periféricos, correspondentes às áreas de esmalte, palitos que sofreram fratura durante o corte foram descartados, somente os palitos íntegros correspondentes às áreas da restauração classe II, foram testados. Para facilitar a localização e melhor visualização dos palitos, a cavidade de classe II, as superfícies oclusal e proximal da restauração foram destacadas com caneta permanente de cor preta (Piloto). Os palitos foram mantidos úmidos, imersos em água destilada à temperatura de $37^{\circ} \mathrm{C}$, em estufa, até o momento da realização do teste de microtração.

\section{Ensaio de Resistência de União - Microtração}

Os espécimes foram fixados pelas suas extremidades ao dispositivo de microtração, semelhante às Garras de Geraldelli, com um gel adesivo à base de cianoacrilato (Super Bond Gel Control - Loctite, Henkel Ltda., SP, Brasil) e adaptados na máquina universal de testes (kratos - modelo IKCL3-USB, Equipamentos Industriais Ltda., Taboão da Serra, SP, Brasil), pertencente ao Centro de Pesquisa em Biomateriais - CPqB da FOP, de modo a posicionar as interfaces adesivas perpendicularmente ao longo eixo da força de tração, submetendo-os ao ensaio mecânico a uma velocidade de $0,5 \mathrm{~mm} / \mathrm{min}$ até a ruptura do espécime, com célula de carga de $20 \mathrm{~kg}$. A máquina de ensaio emitiu os resultados em Newton (N), os quais foram convertidos em Megapascal (MPa), através da divisão do resultado da força (N) pela área da adesão.

Os dados foram analisados descritivamente através das medidas estatísticas: média, desvio padrão e coeficiente de variação, e inferencialmente através do teste F (ANOVA) e no caso de diferença significativa foi utilizada teste de comparações múltiplas de Tukey. A verificação das hipóteses de igualdade de variâncias e de normalidade dos dados foi realizada respectivamente através dos testes $\mathrm{F}$ de Levene e Shapiro-Wilk.

A margem de erro utilizada nas decisões dos testes estatísticos foi de 5\%. Os dados foram digitados na planilha EXCEL e o programa utilizado para obtenção dos cálculos estatísticos foi o SPSS (Statistical Package for the Social Sciences) na versão 21.

\section{Resultados}

Na Tabela 2 pode-se observar que por meio das comparações múltiplas de Tukey se comprova diferença significativa entre: o grupo Surefil SDR Flow com todos os demais grupos e entre X-Tra base com todos, exceto com o grupo da Filtek Flow convencional. Foi utilizada diferença significativa ao nível de 5,0\%. (1): Medidas em Mpa. (2): Através do teste F 
(ANOVA) com comparações do teste de Tukey. Obs: Se todas as letras entre parêntesis são distintas comprova-se diferenças significativas entre os grupos correspondentes.

Tabela 2. Resistência de união à microtração $(\mathrm{RU} \mu \mathrm{T})$ por grupo $(\mathrm{n}=10)$.

\begin{tabular}{lcc}
\hline Grupo & Média $^{(1)}$ & Desvio padrão $^{(1)}$ \\
\hline Filtek Bulk Fill Flow & $26,98^{(\mathrm{D})}$ & 11,28 \\
X-Tra Base & $41,46^{(\mathrm{B})}$ & 14,43 \\
Surefil SDR Flow & $64,37^{(\mathrm{A})}$ & 14,93 \\
Filtek Flow & $36,85^{(\mathrm{DB})}$ & 15,22 \\
Filtek Z 350 & $20,99^{(\mathrm{E})}$ & 9,31 \\
Valor de $p$ & $p^{(2)}<0,001^{*}$ & \\
& & \\
\hline
\end{tabular}

Fonte: Autores.

\section{Discussão}

A hipótese nula não foi aceita, uma vez que as resinas apresentaram diferenças significativas nos resultados encontrados para resistência de união. A resina bulk do tipo flow, Surefil SDR (Dentsply) obteve resultados superiores às demais resinas estudadas, e a resina X-tra Base (Voco) também, exceto quando comparada com a resina Filtek Flow que assim como a Surefil SDR obteve melhor comportamento que a resina composta convencional, Filtek Supreme XT. Tais achados corroboram com (Kumagai et al., 2015), onde a Surefil SDR apresentou valores de resistência de união significativamente maiores do que a Filtek Z350 em cavidades na Classe II MOD com caixas proximais profundas.

O primeiro material disponibilizado no mercado foi a resina Surefil SDR Flow (Dentsply), sendo caracterizada pela elevada translucidez, baixo módulo de elasticidade e alta quantidade de partículas de carga (68\% em peso). Também possui componentes resinosos com grupos fotoativáveis de dimetacrilato de uretano e outros que controlam a cinética de polimerização, atuando como moduladores de contração, reduzindo a tensão geradas durante a fotopolimerização (Czasch \& Ilie, 2013; Nicoleta Ilie \& Hickel, 2010; van Ende et al., 2017).

As resinas bulk fill não se diferenciam em sua composição química básica das resinas compostas convencionais nanohíbridas e microhíbridas. Sua matriz orgânica contém monômeros como Bis-GMA, UDMA, TEGDMA, EBPDMA e outros, assim como sua carga inorgânica constituída por partículas já conhecidas (El-Safty et al., 2012; N. Ilie et al., 2013; Nicoleta Ilie \& Hickel, 2010). Como característica das resinas bulk pode-se ressaltar um aumento da translucidez, que contribui e muito para ampliar sua profundidade de polimerização, por permitir maior passagem da luz através do incremento único de 4-5 mm, apresentando assim, propriedades satisfatórias relacionadas ao grau de conversão, dureza vickers, contração de polimerização e tensões de contração de polimerização (Lee, 2008; Zorzin et al., 2015). As alterações no índice de refração da luz entre matriz orgânica e a fase inorgânica, as dimensões e tipo de partículas de carga, os pigmentos e os opacificadores também influenciam a translucidez (Bucuta \& Ilie, 2014; Flury et al., 2014; Fronza et al., 2017; Par et al., 2018). No presente estudo, a fotopolimerização das restaurações foi realizada com luz LED monowave em modo contínuo com $1200 \mathrm{~mW} / \mathrm{cm}^{2}$ de intensidade da luz. Diversos estudos avaliaram a capacidade da canforoquinona (CQ) sensibilizados pela faixa de emissão de luz utilizada (Rocha et al., 2017).

A qualidade da adesão de materiais resinosos na região de dentina cervical de cavidades classe II pode ser crítica, onde as tensões associadas à contração de polimerização podem ser maiores. A técnica de inserção incremental é preconizada para minimizar esses problemas (Park et al., 2008; van Dijken \& Pallesen, 2015). Diversos estudos com diferentes 
metodologias tem sido realizados para evidenciar a qualidade adesiva dos materiais resinosos. Estudos em tomografia de coerência óptica mostraram desempenho melhor da Surefil SDR Flow quando comparados aos compósitos convencionais, apresentando redução da contração de polimerização volumétrica em $20 \%$ através de micro tomografia computadorizada realizada em cavidades classe I (Hirata et al., 2015; Nazari et al., 2013). Como restaurações de resina composta podem gerar tensões na interface adesiva compósito/substrato dental, a compreensão e acompanhamento da evolução temporal desta resistência de união deve permitir uma melhor avaliação da longevidade e qualidade das restaurações adesivas (Guo et al., 2016). Porém devemos considerar resultados diferentes de acordo com a região da dentina ou esmalte está sendo realizado o estudo, pois uma variabilidade substancial deve ser esperada.

Tauböck (2019) utilizando um espectrômetro infravermelho (Cary 630 FTIR; Agilent Technologies, Santa Clara, CA, EUA) avaliou o grau de conversão de resinas compostas de baixa e alta viscosidade, a resina bulk SDR entre os compostos de resina fluídos testados apresentou valores maiores de conversão e menor estresse de contração proveniente da fotoativação corroborando com os achados deste estudo. O mesmo foi observado por (Rizzante et al., 2019), que ao realizar o teste de tensão de contração, mostrou que a Surefil SDR obteve valores significativamente menores de tensão de contração de polimerização quando comparada à resina composta convencional Filtek Z350XT.

Gezawi et al (2015) analisaram in vitro a integridade marginal da cervical de cavidades classe II restauradas com resinas bulk fill, técnica incremento único versus resinas convencionais, técnica incremental, após ciclagem termomecânica usando digitalização réplica em microscopia eletrônica de varredura (MEV), posteriormente classificadas de acordo com critérios FDI, demostrando que a integridade marginal não foi significativamente influenciada pelo uso de resinas bulk fill, técnica adesiva, ou pela variação da localização nas margens cervicais (Gezawi, 2015).

Uma das explicações para os bons resultados de resistência de união das resinas bulk fill seria sua capacidade de aliviar as tensões internas antes de atingir o ponto gel devido a moduladores de polimerização. O modulador de polimerização é um grupo fotoativo, quimicamente inserido no metacrilato à base de uretano, que interage com a canforoquinona. Tal interação pode induzir a um desenvolvimento mais lento do módulo de elasticidade, o que permite a redução das tensões geradas pela contração de polimerização, sem afetar a taxa de polimerização e o grau de conversão (Giovannetti et al., 2012).

Tem sido observado desde 1984, que a velocidade da reação de polimerização, também contribui para modular a tensão de contração. A fotoativação mais lenta permite escoamento viscoso e/ou maior relaxamento das cadeias, acomodandoas durante a contração e reduzindo assim as tensões (Davidson et al., 1984). A Surefil SDR apresenta uma polimerização por radicais livres mediado por um cromóforo/modulador que torna o processo de polimerização mais lento, estendendo a fase prégel permitindo uma contração espontânea, mantendo um baixo módulo de elasticidade, que resulta na diminuição das tensões de polimerização na matriz orgânica (Brinkgreve, 2011; Ferracane, 2005). Tal processo pode justificar em parte, os elevados valores de resistência de união da Surefil SDR encontrados neste estudo, assim como os de todas as resinas bulk fill flow, inclusive a resina convencional flow terem apresentado valores superiores à resina Filtek Z350 (controle convencional de média viscosidade), que possui maior módulo de elasticidade (Ferracane, 2005).

O acompanhamento de 3 anos, de ensaio clínico randomizado mostrou bom desempenho da resina Surefill SDR com técnica de incremento único quando comparada com técnica incremental realizada com resina convencional Ceram X mono (Dentsply) (van Dijken \& Pallesen, 2015; Van Dijken \& Pallesen, 2014). Este mesmo estudo após 5 anos de acompanhamento a resina bulk fill (SDR) mostrou desempenho um pouco melhor, mas não estatisticamente significativa, em comparação com a técnica incremental convencional em restauração classe II (van Dijken \& Pallesen, 2016). Ensaios clínicos randomizados com acompanhamento de 36 meses com compósito resinosos não encontraram diferenças significativas na integridade marginal de restaurações realizadas com resina Bulk Fill quando comparadas com resinas compostas, confirmando que ambos compósitos tem bom desempenho clínico em restaurações posteriores (Loguercio et al., 2019; Moura et al., 2021). 
A revisão sistemática e meta-análise de Mai M. Akah et al, (2016), relatou que as resinas compostas bulk fill, poderiam resolver substancialmente os problemas inerentes às resinas compostas convencionais como a contração de polimerização e ao grau de conversão, especialmente quando usadas em cavidades profundas de dentes posteriores sem prejudicar a sua resistência de união à dentina (Akah et al., 2016). Entretanto a revisão sistemática e meta-análise de Veloso et al (2019), destacou o desempenho clínico semelhante tanto para resinas compostas bulk-fill, como para as convencionais ao longo de um período de acompanhamento de 12 a 72 meses (S et al., 2019).

No presente estudo foi avaliado o tipo de fratura, devido à perda de corpos de prova, o que inviabilizou esta análise. Outra limitação deste estudo, que pode ser superada em outras pesquisas, é a realização da coleta da idade dos pacientes que tiverem seus dentes extraídos e utilizados no teste de microtração, podendo ser discutido se a idade da dentina terá influência nos resultados. Estudos clínicos correspondem ao teste final dos materiais e longevidade das restaurações, desse modo, sua execução é essencial para maior clareza do desempenho de determinado material, e/ou técnica. Os estudos in vitro tem sua importância e são necessários, mas apenas estimam o possível comportamento clínico dos materiais, não podem ser determinantes em recomendar ou não certo material.

\section{Conclusão}

De acordo com a metodologia empregada e com base nos resultados obtidos, conclui-se que além de contribuir para otimização e simplificação da técnica restauradora, as resinas bulk fill de baixa viscosidade inseridas em incremento único, demonstraram resistências de união satisfatória na parede cervical de cavidades Classe II, destacando-se a resina Surefil SDR.

\section{Referências}

Akah, M. M., Daifalla, L. E., \& Yousry, M. M. (2016). Bonding of bulk fill versus contemporary resin composites: A systematic review and meta-analysis. Indian Journal of Science and Technology, 9(20), 8-11.

Brinkgreve, R. B. . (2011). Scientific Manual. Plaxis.

Bucuta, S., \& Ilie, N. (2014). Light transmittance and micro-mechanical properties of bulk fill vs. conventional resin based composites. Clinical Oral Investigations, 18(8), 1991-2000.

Chesterman, J., Jowett, A., Gallacher, A., \& Nixon, P. (2017). Bulk-fill resin-based composite restorative materials: A review. British Dental Journal, 222(5), $337-344$.

Czasch, P., \& Ilie, N. (2013). In vitro comparison of mechanical properties and degree of cure of bulk fill composites. Clinical Oral Investigations, 17(1), $227-235$.

Davidson, C. L., de Gee, A. J., \& Feilzer, A. (1984). The Competition between the Composite-Dentin Bond Strength and the Polymerization Contraction Stress. Journal of Dental Research, 63(12), 1396-1399.

Durão, M. de A., Andrade, A. K. M. de, Santos, M. do C. M. da S., Montes, M. A. J. R., \& Monteiro, G. Q. de M. (2020). Clinical Performance of Bulk-Fill Resin Composite Restorations Using the United States Public Health Service and Federation Dentaire Internationale Criteria: A 12-Month Randomized Clinical Trial. European Journal of Dentistry.

El-Safty, S., Silikas, N., \& Watts, D. C. (2012). Creep deformation of restorative resin-composites intended for bulk-fill placement. Dental Materials, 28(8), 928-935.

Ferracane, J. L. (2005). Developing a more complete understanding of stresses produced in dental composites during polymerization. Dental Materials, 21(1), $36-42$.

Ferracane, J. L. (2011). Resin composite - State of the art. Dental Materials, 27(1), 29-38.

Flury, S., Peutzfeldt, A., \& Lussi, A. (2014). Influence of increment thickness on microhardness and dentin bond strength of bulk fill resin composites. Dental Materials, 30(10), 1104-1112. https://doi.org/10.1016/j.dental.2014.07.001

Fronza, B. M., Ayres, A. P. A., Pacheco, R. R., Rueggeberg, F. A., Dias, C. T. S., \& Giannini, M. (2017). Characterization of inorganic filler content, mechanical properties, and light transmission of bulk-fill resin composites. Operative Dentistry, 42(4), 445-455.

Gezawi, M. El. (2015). Marginal Integrity of Bulk Versus Incremental Fill Class II Composite Restorations.

Giovannetti, A., Goracci, C., Vichi, A., Chieffi, N., Polimeni, A., \& Ferrari, M. (2012). Post retentive ability of a new resin composite with low stress 
behaviour. Journal of Dentistry, 40(4), 322-328.

Guo, J., Holmes, B., Yang, B., Li, Y., Heo, Y. C., Chen, J., \& Fok, A. (2016). Determining the temporal development of dentin-composite bond strength during curing. Dental Materials, 32(8), 1007-1018.

Hirata, R., Kabbach, W., De Andrade, O. S., Bonfante, E. A., Giannini, M., \& Coelho, P. G. (2015). Bulk Fill Composites: An Anatomic Sculpting Technique. Journal of Esthetic and Restorative Dentistry, 27(6), 335-343.

Ilie, N., Bucuta, S., \& Draenert, M. (2013). Bulk-fill resin-based composites: An in vitro assessment of their mechanical performance. Operative Dentistry, $38(6), 618-625$.

Ilie, Nicoleta, \& Hickel, R. (2010). Investigations on a methacrylate-based flowable composite based on the SDR TM technology. Dental Materials, 27(4), $348-355$.

Kumagai, R. Y., Zeidan, L. C., Rodrigues, J. A., Reis, A. F., \& Roulet, J. F. (2015). Bond strength of a flowable bulk-fill resin composite in Class II MOD cavities. The Journal of Adhesive Dentistry, 17(5), 427-432.

Lazarchik, D. A., Hammond, B. D., Sikes, C. L., Looney, S. W., \& Rueggeberg, F. A. (2007). Hardness comparison of bulk-filled/transtooth and incrementalfilled/occlusally irradiated composite resins. Journal of Prosthetic Dentistry, 98(2), 129-140.

Lee, Y. K. (2008). Influence of filler on the difference between the transmitted and reflected colors of experimental resin composites. Dental Materials, 24(9), $1243-1247$.

Li, X., Pongprueksa, P., Van Meerbeek, B., \& De Munck, J. (2015). Curing profile of bulk-fill resin-based composites. Journal of Dentistry, 43(6), 664-672.

Loguercio, A. D., Rezende, M., Gutierrez, M. F., Costa, T. F., Armas-vega, A., \& Reis, A. (2019). Randomized 36-month follow-up of posterior bulk- fi lled resin composite restorations. Journal of Dentistry, 85(May), 93-102.

Moura, A. G. dos S. M., Melo, M. G. R. R. de, Santos, W. T. do N. P., Cassiano Alves Bezerra, A. L., Monteiro, G. Q. de M., \& De Almeida Durão, M. (2021). Avaliação in vitro da estabilidade de cor de resinas compostas bulk-fill. Archives of Health Investigation, 10(4), 530-535.

NASCIMENTO, A. S., LIMA, E. A. de, DURÃO, M. de A., SOUSA, Y. de C., CORREIA, T. C., \& BRAZ, R. (2016). Marginal microleakage in Bulk Fill resins. Revista de Odontologia Da UNESP, 45(6), 327-331.

Nayif, M. M., Nakajima, M., Foxton, R. M., \& Tagami, J. (2008). Bond strength and ultimate tensile strength of resin composite filled into dentine cavity; effect of bulk and incremental filling technique. Journal of Dentistry, 36(3), 228-234.

Nazari, A., Sadr, A., Saghiri, M. A., Campillo-Funollet, M., Hamba, H., Shimada, Y., Tagami, J., \& Sumi, Y. (2013). Non-destructive characterization of voids in six flowable composites using swept-source optical coherence tomography. Dental Materials, 29(3), 278-286.

Par, M., Repusic, I., Skenderovic, H., Klaric Sever, E., Marovic, D., \& Tarle, Z. (2018). Real-time light transmittance monitoring for determining polymerization completeness of conventional and bulk fill dental composites. Operative Dentistry, 43(1), 19-31.

Park, J., Chang, J., Ferracane, J., \& Lee, I. B. (2008). How should composite be layered to reduce shrinkage stress: Incremental or bulk filling? Dental Materials, 24(11), 1501-1505.

Rizzante, F. A. P., Mondelli, R. F. L., Furuse, A. Y., Borges, A. F. S., Mendonça, G., \& Ishikiriama, S. K. (2019). Shrinkage stress and elastic modulus assessment of bulk-fill composites. Journal of Applied Oral Science, 27, 1-9.

Rocha, M. G., De Oliveira, D. C. R. S., Correa, I. C., Correr-Sobrinho, L., Sinhoreti, M. A. C., Ferracane, J. L., \& Correr, A. B. (2017). Light-emitting diode beam profile and spectral output influence on the degree of conversion of bulk fill composites. Operative Dentistry, 42(4), 418-427.

Rosatto, C. M. P., Bicalho, A. A., Veríssimo, C., Bragança, G. F., Rodrigues, M. P., Tantbirojn, D., Versluis, A., \& Soares, C. J. (2015). Mechanical properties, shrinkage stress, cuspal strain and fracture resistance of molars restored with bulk-fill composites and incremental filling technique. Journal of Dentistry, 43(12), 1519-1528.

S, V., Lemos C, S, dE M., do Egito Vasconcelos B, E, P., \& G, M. (2019). Clinical performance of bulk-fill and conventional resin composite restorations in posterior teeth: a systematic review and meta-analysis. Clinical Oral Investigations, 23(1), 221-233.

van Dijken, J. W. V., \& Pallesen, U. (2015). Randomized 3-year clinical evaluation of class I and II posterior resin restorations placed with a bulk-fill resin composite and a one-step self-etching adhesive. The Journal of Adhesive Dentistry, 17(1), 81-818.

van Dijken, J. W. V., \& Pallesen, U. (2016). Posterior bulk-filled resin composite restorations: A 5-year randomized controlled clinical study. Journal of Dentistry, 51, 29-35.

Van Dijken, J. W. V., \& Pallesen, U. (2014). A randomized controlled three year evaluation of bulk-filled posterior resin restorations based on stress decreasing resin technology. Dental Materials, 30(9), e245-e251.

van Ende, A., de Munck, J., Lise, D. P., \& van Meerbeek, B. (2017). Bulk-fill composites: A review of the current literature. Journal of Adhesive Dentistry, 19(2), 95-109.

Yazici, A. R., Antonson, S. A., \& Kutuk, Z. B. (2017). Thirty-Six-Month Clinical Comparison of Bulk Fill and Nanofill Composite Restorations. 478-485.

Zaruba, M., Wegehaupt, F. J., \& Attin, T. (2013). Comparison between different flow application techniques: SDR vs flowable composite. Journal of Adhesive Dentistry, 15(2), 115-121.

Zorzin, J., Maier, E., Harre, S., Fey, T., Belli, R., Lohbauer, U., Petschelt, A., \& Taschner, M. (2015). Bulk-fill resin composites: Polymerization properties 
Research, Society and Development, v. 10, n. 11, e213101118970, 2021

(CC BY 4.0) | ISSN 2525-3409 | DOI: http://dx.doi.org/10.33448/rsd-v10i11.18970

and extended light curing. Dental Materials, 31(3), 293-301. 